\section{$\S 13$. Three-Dimensional Particle-In-Cell Simulation of Scrape-off-Layer Plasmas}

Ishiguro, S.

There is a growing interest in investigation of three-dimensional kinetic effects of scrape off layer plasmas in magnetic fusion devices. For example, charged particle dynamics not only along a magnetic field but also across a magnetic field plays an important role in a blob transport across a magnetic field in a scrape off layer ${ }^{1.5}$. Plasma behavior in the helical diverter in Large Helical Device (LHD) also may cast an importance of three dimensional kinetic treatments. With this situation in mind, we have developed three dimensional Particle-In-Cell (PIC) simulation code for investigation of dynamic plasma behavior in a scrape-off-layer.

Figure 1 shows simulation configuration. Particle absorbing boundaries corresponding to diverter plates are placed in the ends of z-direction. Particle absorbing boundary is also placed at one end of the $\mathrm{x}$-direction. This corresponds to a first wall. A particle impinging to particle absorbing boundaries is removed from the system. The particle absorbing boundaries are grounded. On the other hand, periodic boundary condition is applied in the y-direction. A uniform external magnetic field is pointing into the $\mathrm{z}$-direction.

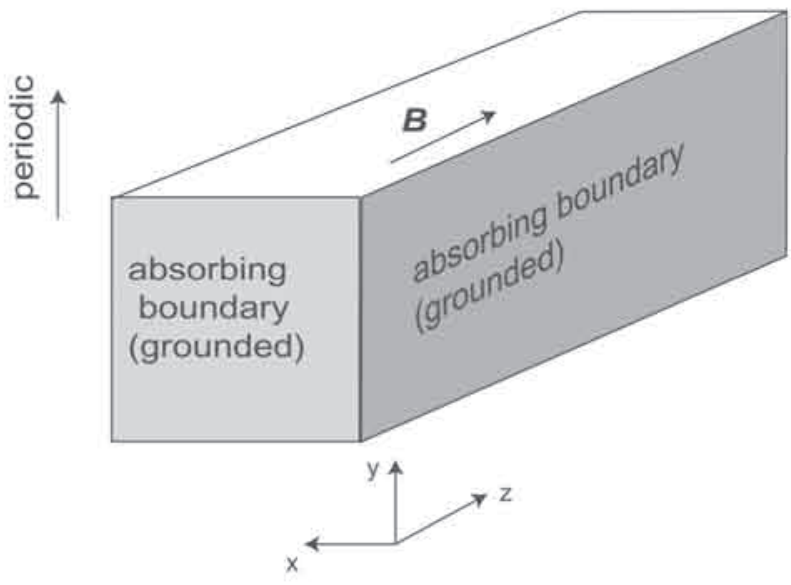

Fig. 1. Configuration of three dimensional Particle-In-Cell simulation for investigation of scrape off layer plasmas.

In order to investigate physics of plasmas in a scrape-off layer, large spatial scale and long time scale simulations are necessary. So we have to make use of a large number of CPUs and Large distributed memories.

The simulation code is constructed by using High Performance FORTRAN (HPF) and is arranged to have a good performance at the National Institute for Fusion Science (NIFS) "Plasma Simulator" NEC SX-7/160M5 system which consists of five nodes, 160 processor elements, 1.28TBytes memory, vector parallel super computer. The peak peak performance is 1.44 TFloaps. The parallelization in each node is done by auto-parallelization and the parallelization among multi nodes is done by using HPF. The base code with periodic boundary condition has attained 224GFlops.

We have made some preliminary runs to check the basic property of plasma surrounded by particle absorbing boundaries. Initially, Maxwellian electrons and ions are uniformly distributed in the system. Simulation parameters are following: the system lengths $L_{x}=L_{y}=128 \lambda_{D e}$, and $\mathrm{L}_{2}=1024 \lambda_{\mathrm{Dx}}$, the grid cell number $128 \times 128 \times 1024$, the temperature ration $T / T=1$, the ion to electron mass ration $\mathrm{m} / \mathrm{m}_{\mathrm{c}}=400$, the electron cyclotron frequency to electron plasma frequency $\omega_{c c} / \omega_{\mathrm{pe}}=2$, the number of simulation particles $\mathrm{N}_{\mathrm{i}}=\mathrm{N}_{\mathrm{e}}=1,073,741,824$. Since escape of electrons from the system to the boundaries is faster than that of ions, plasma region is positively charged. Ion sheaths are created in front of the boundaries at the ends of z-direction. These futures are clearly shown in Fig. 2, which is a potential profile in the $\mathrm{x}-\mathrm{z}$ plane.

This simulation model will be extended to include non-uniform magnetic field and the transport of blobs will be investigated.
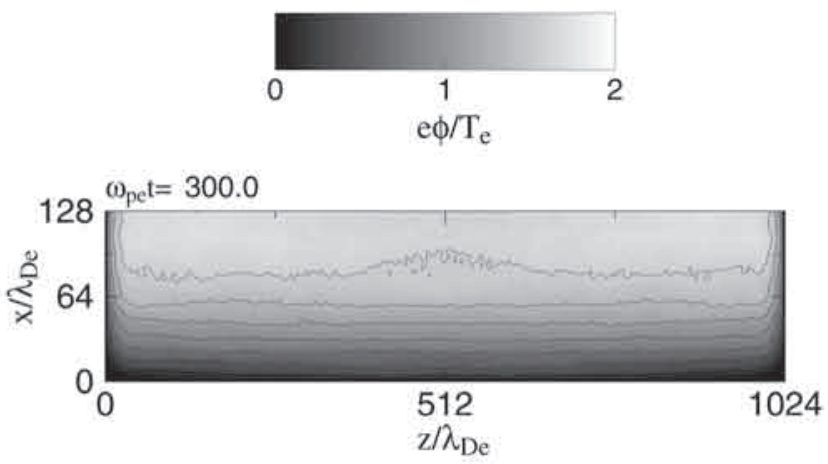

Figure 2: Potential profile in x-z plane.

References

[1] M. Umansky, S. I. Krasheninnikov, B. LaBombard, J. L. Terry, Phys. Plasmas 5, 3373 (1998) .

[2] R. Schneider et al., in: $17^{\text {th }}$ International Conference on Plasma Physics and Controlled Fusion Research, Yokohama, Japan, 19-24 October, 1998, paper FI-CN-69/THP2/05.

[3] B. Lipschultz et al. in: $18^{\text {th }}$ IAEA Fusion Energy Conference, Sorrento, Italy, 4-10 October, 2000, paper IAEA-CN-77/EX5/6.

[4] S. J. Zweben, R. J. Maqueda, D. P. Stotler et al., Nucl. Fusion 44, 134 (2004).

[5] S. I. Krashennnikov, Phys. Lett. A 283, 368 (2001). 